\title{
Potential of Peptide-Based Non-Structural Protein 1 (NS1) Inhibitor in Obstructing Dengue Virus (DENV) Replication
}

\author{
Muhammad Mikail Athif Zhafir Asyura ${ }^{1 *}$, Ahmad Fauzi ${ }^{2}$, Fakhru Adlan Ayub ${ }^{3}$ \\ ${ }^{1}$ Faculty of Medicine, University of Indonesia, Jl. Salemba Raya No.4, Jakarta, Indonesia \\ ${ }^{2}$ Faculty of Medicine, University of Indonesia, Jl. Salemba Raya No.4, Jakarta, Indonesia \\ ${ }^{3}$ Faculty of Medicine, University of Indonesia, Jl. Salemba Raya No.4, Jakarta, Indonesia \\ *Corresponding Author. E-mail: muhammad.mikail91@ui.ac.id Telp: +62-8118884480
}

\begin{abstract}
Introduction: Dengue Virus (DENV) is the pathogen for human dengue fever and is responsible for 390 million infections per year. The viral genome produces about 10 viral protein products, one of them being NS1. The NS1 protein plays a key role in viral replication and stimulation of humoral immune cells, thus being the perfect candidate to create an effective antiviral drug or vaccine for dengue

Methods: Dengue Virus (DENV) is the pathogen for human dengue fever and is responsible for 390 million infections per year. The viral genome produces about 10 viral protein products, one of them being NS1. The NS1 protein plays a key role in viral replication and stimulation of humoral immune cells, thus being the perfect candidate to create an effective antiviral drug or vaccine for dengue

Conclusion: The review established promising results of using peptide-based intervention on NS1. Further in vivo and randomized controlled trials are advised to solidify the applicability and biosafety of the intervention
\end{abstract}

Keywords: antiviral agents; dengue; peptides; proteins
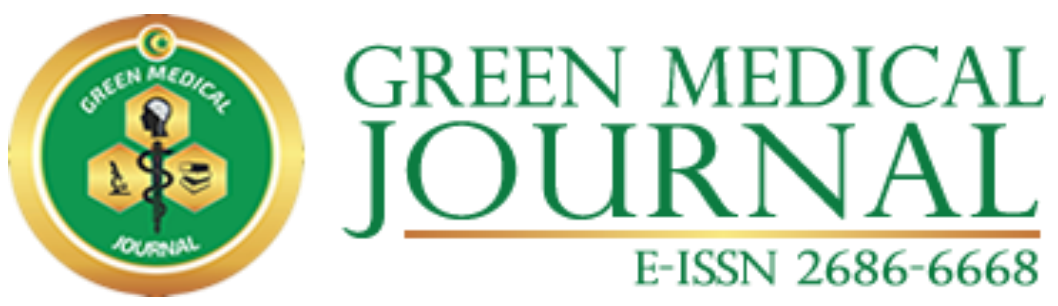

Article history:

Received: 12 February 2021 Accepted: 26 April 2021

Published: 30 April 2021

Published by:

Faculty of Medicine

Universitas Muslim Indonesia

Mobile number:

+6282197210007
Address:

J1. Urip Sumoharjo Km. 5, Makassar

South Sulawesi, Indonesia

Email:

greenmedicaljournal@umi.ac.id 


\section{Introduction}

Dengue Virus (DENV) is a single stranded RNA flavivirus and a major causative agent of dengue fever, West Nile, and yellow fever. ${ }^{[1]}$ DENV is transmitted via mosquito (aedes aegypti and aedes albopictus) saliva to humans. Throughout history, DENV is responsible for 390 million infections per year with 96 million among them showing clinical symptoms and $2.5 \%$ mortality rate each year. ${ }^{[2]}$ DENV infections are more common in tropical countries as in Indonesia, the prevalence of DENV infections are 77.96 cases per 100,000 person-years in 2016. ${ }^{[2]}$

Dengue was a global health issue that endemic in around 100 countries, mostly tropical and subtropical. Over the last decade, Dengue issues have increased quickly. WHO states that dengue infects about 390 million and causes up to 100 million cases every year. WHO proposed a new dengue classification based on the severity because of changes in epidemiology and incidence rates. ${ }^{[2]}$ Indonesia is one of endemic countries and the epidemiology of dengue fever often shows as a case series. Indonesia has the second largest dengue fever cases among 30 other endemic countries and the most prevalent in Java, particularly West, Central, and East Java. ${ }^{[1,2]}$

DENV has 4 different serotypes: DENV-1, DENV-2, DENV-3, and DENV-4. ${ }^{[3]}$ The DENV RNA strain contains 10 viral protein products that can be classified into 3 structural proteins and 7 non-structural proteins. The 3 structural protein classes are Envelope (E), Capsid (C), and pre-Membrane (prM). ${ }^{[1-5]}$ The other 7 nonstructural (NS) proteins are NS1, NS2A, NS2B, NS3, NS4A, NS4B, and NS5. ${ }^{[6-10]} \mathrm{NS} 1$ is a major protein in DENV viral replication ${ }^{[5,9]} \mathrm{NS} 1$ is sequenced by 352 amino acid residue and has $70 \%$ similarity to other DENV serotype. ${ }^{[5]}$ Other roles of NS1 include stimulating immune cell to release vasoactive cytokines which lead to vascular leakage. ${ }^{[1,10]}$ NS1 protein has 3 important domains: the hydrophobic $\beta$-roll as a domain for dimerization process (located on 1-29 residue), the wing as a connector subdomain (located on residue number $30-37$ and $152-180$, the $\alpha / \beta$ subdomain and residues number $38-151$ ), and $\beta$-ladder (residue number 181-352) visible in Figure 1. Which play a vital role in NS1 physiology. ${ }^{[1,10]}$ This type of protein has been considered as a potential vaccine of dengue due to its pathological roles. NS1 can be soluble in host plasma and bind to prothrombin, then alter its function. Also, NS1 can bind to TLR4, TLR2, and TLR6 Receptor which induce proinflammatory cytokines release, leading to vascular leakage. The anti-NS1 vaccines act as an antagonist of this NS1 protein to inhibit its pathological roles. Also, anti-NS1 can induce antibodies and complement-dependent mechanism to lysis the DENV-infected cells. ${ }^{[6-8]}$

Many researches have been conducted to search for DENV vaccines or drugs. ${ }^{[11-16]}$ Nevertheless, there are still no effective and specific vaccines or drugs to treat DENV infection, implied by the ever-increasing prevalence of DENV worldwide and also in Indonesia. ${ }^{[2,11,13-16]}$. On the other hand, data shows that dengue fever prevalence is very high and keeps rising especially in Asia. ${ }^{[2]}$ Treatment for dengue fever and severe 
dengue fever are focused on maintaining and controlling patient body fluid. ${ }^{[2,17]}$ Therefore, to control and prevent DENV infection more effectively, research for DENV vaccines or drugs is still one of major focus.

Understanding which type of vaccines and the mechanism of action remained a big hurdle for scientists. In this literature review, we focused on reviewing the action mechanism of NS1 and DENV outcome if the NS1 protein is inhibited. Despite much research, there is still no exact mechanism of NS1 in viral replication. Although a study suggests that NS1 plays a role in formation and stabilization of viral membrane organelles. ${ }^{[2,6-8]}$ By understanding these NS1 mechanisms, scientists have found some potential candidates for vaccines. Therefore, these vaccines still need to be investigated to improve it is efficacy and safety. ${ }^{[9]}$ Hence, by considering the minimal and slow process in DENV vaccine development, peptide-based inhibition of NS1 protein could play a major role in decreasing DENV numbers significantly. Thus, we conducted this literature review based on that purpose in mind and to contribute towards the World Health Organization's sustainable development goals (SDG) number 3, which is good health and wellbeing. Especially two of its targets to reduce mortality of communicable disease and cure all preventable causes of deaths in under 5 years old children as in Indonesia itself, 18.01\% dengue cases had been reported with death proportion of $38.89 \%$ among all ages per December 2020. ${ }^{[15]}$

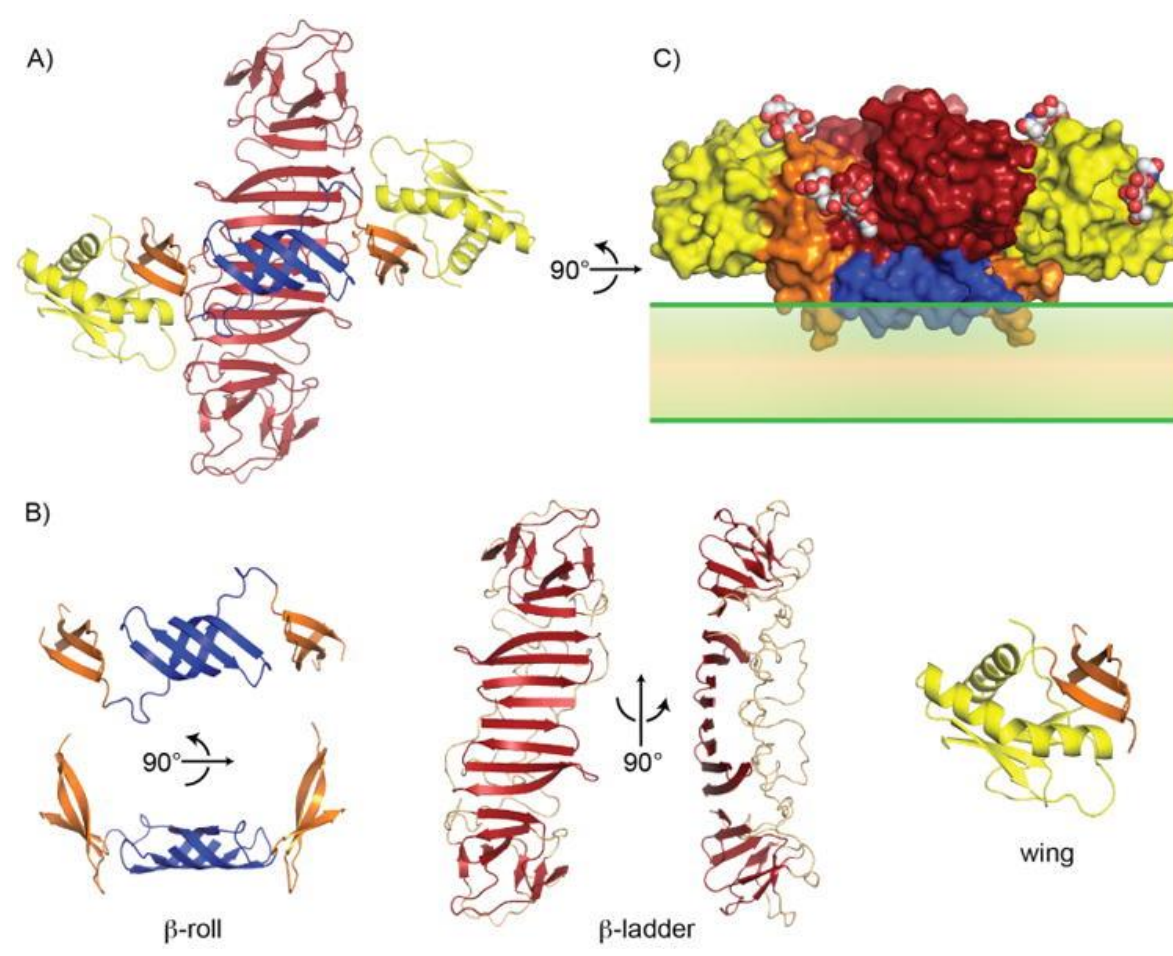

Figure 1. NS-1 Protein Structure ${ }^{[9]}$

\section{Content}

Search Strategy

This literature review was assembled by searching, compiling, and analyzing various studies that 
Green Medical Journal Vol.3 Issue: 1 (April, 2021)

e-ISSN: 2686-6668

investigated the role of non-structural protein 1 (NS1) in viral replication and the potential use of peptidebased treatment that inhibits the protein. Search terms used included ("Peptide" OR "Peptide drug") AND ("Nonstructural protein 1" OR "NS1") AND ("Dengue") AND ("Inhibit" OR "Reduce"). The sources were cited from Pubmed/Medline, Science Direct, Proquest, Wiley Online Library, Directory of Open Access Journal (DOAJ), and Cochrane library. From the databases, 11 studies were included in this review in which 9 assessed the potential of NS1 as a target and 2 studies assessed the potential usage of peptide-based interventions to inhibit DENV replication. Variables mentioned and analyzed from the 9 included studies for NS1 targeting includes the mechanism and the advantages if compared to the current and other alternative interventions. Furthermore, analysis of the 2 included studies for DENV inhibition will focus on the efficacy of the said treatment on in vitro and in vivo situations. Additional materials collected via individual searching from the said databases was also conducted when necessary, to supplement this review

Figure 2. Provides a visual representation of the literature search strategy

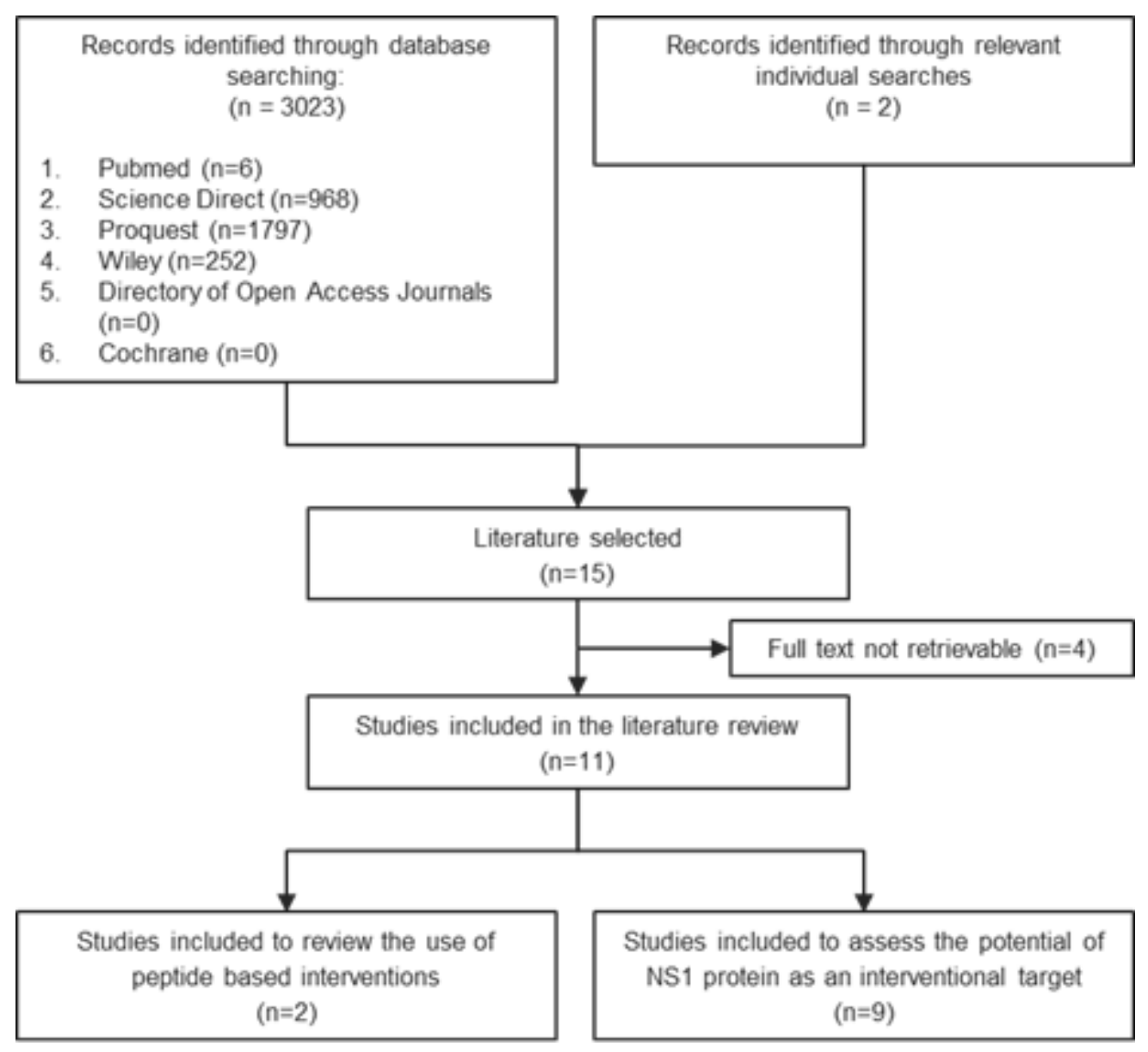

Figure 2. Flowchart of the Literature Search Strategy

\section{Current State of Dengue}

The graph below (Figure 3) shows the Incidence Rate of Dengue per 100.000 populations in Indonesia bv Province in 2016. From the graph. we can conclude Bali has the highest Incidence Rate and the lowest is 
Papua. The first case of dengue fever was in Jakarta, 1968. Since then, the Incidence Rate (IR) of dengue fever shows an increasing trend. It increased from 0.05 - 35-40 per 100.000 population in 2013. Dengue fever has infected all of 34 provinces and $80 \%$ of 497 cities in 2017. The spreading in the cities of Indonesia can be looked at from the incidence rate per 100.000 population in the cities from each year, and it shows positive trends. These are summarized on graph in Figure 4 that shows positive trends of incidence rate per 100.000 population from $1986-2016 .^{[15]}$

IR/100 000

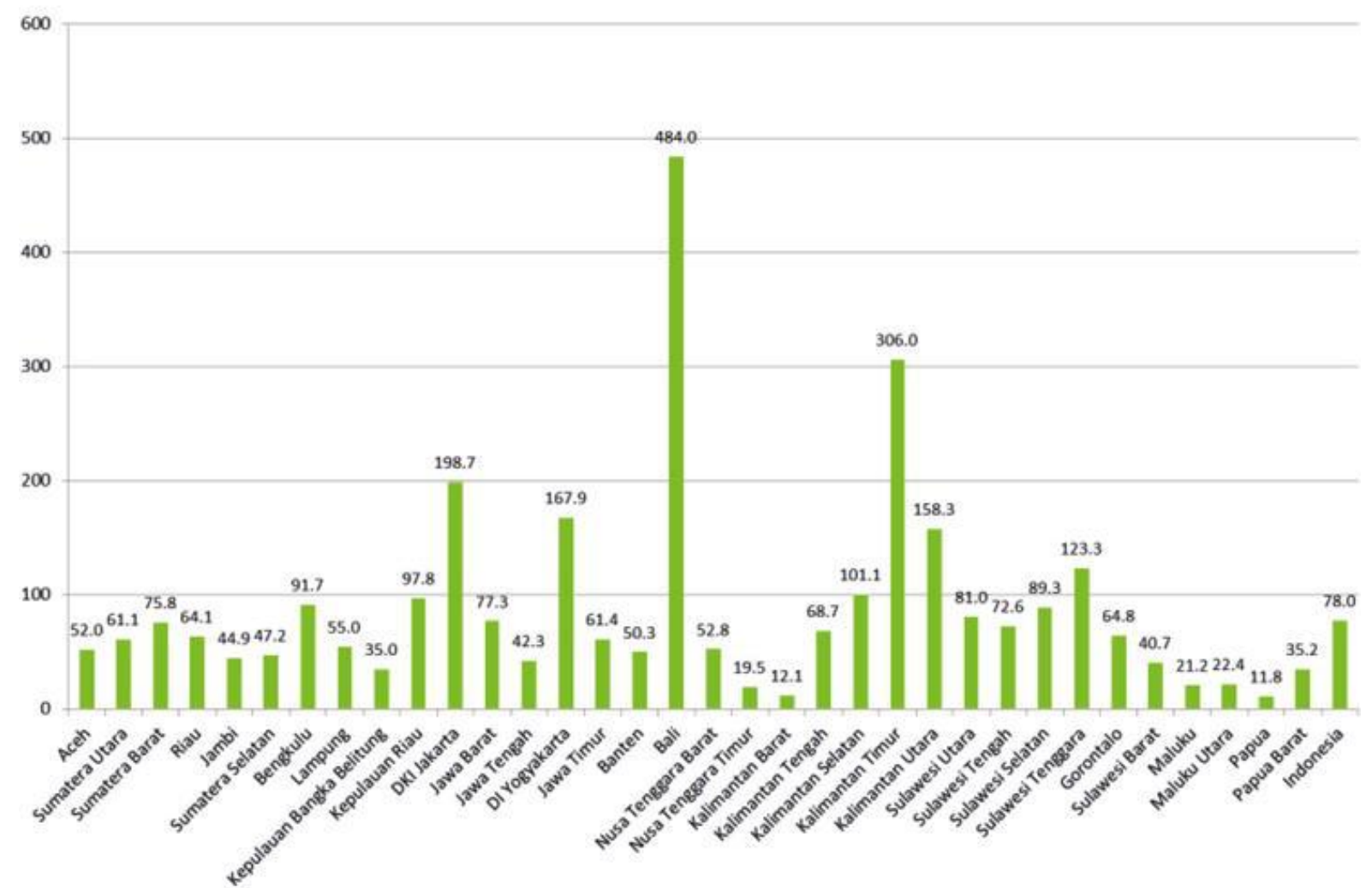

Province

Figure 3. Incidence Rate of Dengue in Indonesia by Province ${ }^{[15]}$

Vaccine for dengue is still an issue to this day because there is no effective or commercial vaccine. ${ }^{[10]}$ The first dengue vaccine introduced is Dengvaxia developed by Sanofi Pasteur. The vaccine licensed in December 2015 in Mexico for individuals between 9-45 years of age. ${ }^{[6-8]}$ Many approaches have been used, such as live attenuated, DNA Vaccine, subunit antigen, and other approaches, and several groups show good progress and efforts in vaccine development. Currently, the strategy has each status and phase, as shown in the table below. ${ }^{[15]}$ 


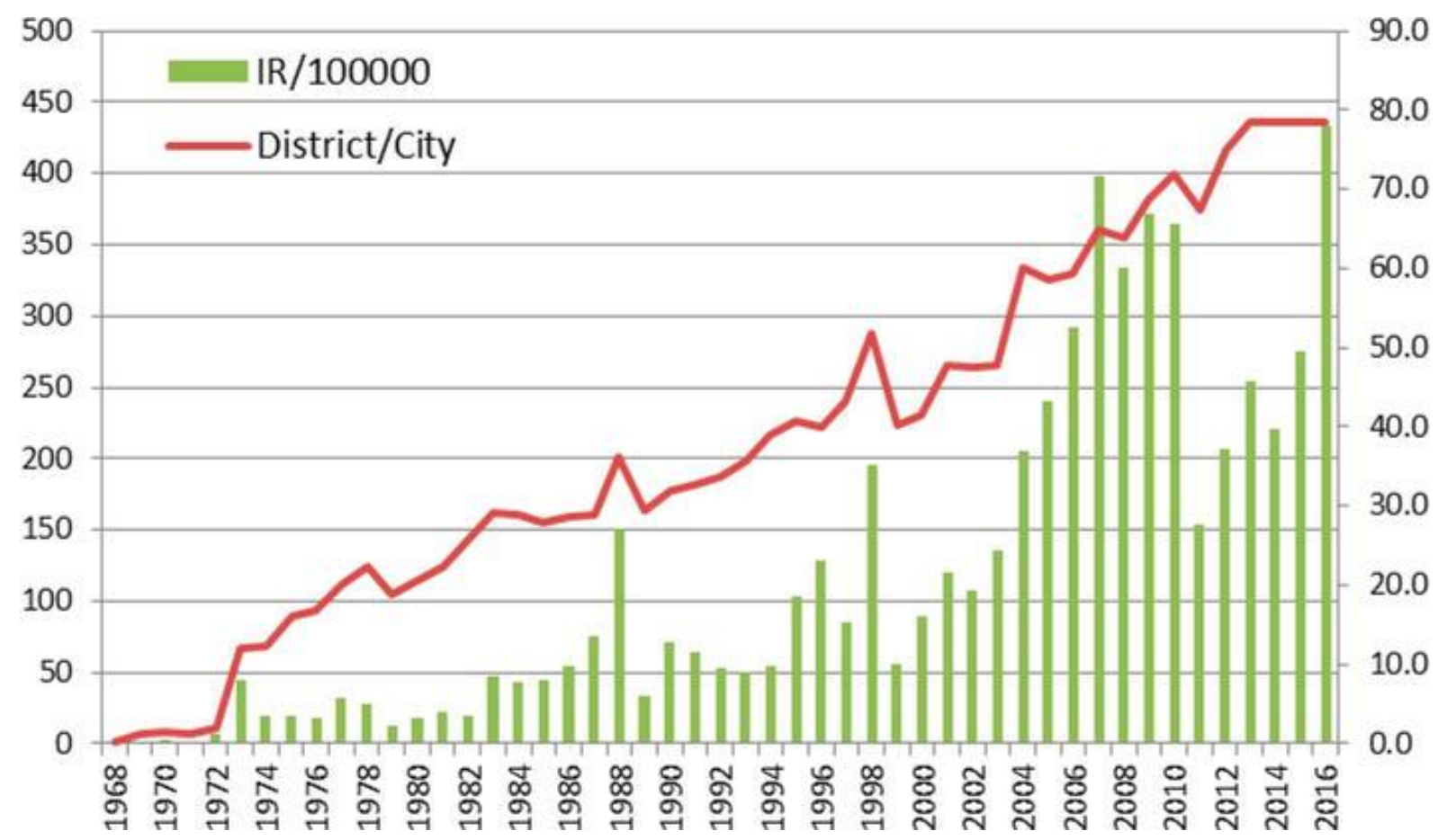

Figure 4. Incidence Rate of Dengue in Indonesia and Number of Cities Infected ${ }^{[15]}$

\section{The Importance of Non-Structural Protein 1 (NS1)}

As mentioned before, no such anti-dengue drug or vaccine has been successfully synthesized and was proven effective against the viral strain despite its increasing need and urgency. Connected to that concern, increasing focus is needed to be put on modalities other than supportive care. Antiviral approaches have been shown to target structural and non-structural proteins of DENV due to their pivotal role in viral replication and transmission. Multiple candidate proteins have been identified with NS proteins being the majority, especially the NS1 protein. ${ }^{[18-23]}$ The DENV NS1 protein is a glycoprotein translated by the viral genome in different structural forms (Figure 1.) depending on its location. The NS1 protein is expressed in the form of a monomer but transforms into a membrane-bound dimer on the cell surface of the host cell. Moreover, the protein is secreted in the form of a hexamer (open barrel structure with three dimers forming a hydrophobic region) that cycles in the patient's circulatory system. ${ }^{[21,23]}$ Functionality wise, the protein plays an essential role in viral replication but the exact mechanism is still unknown. Study conducted by Plaszczyca et al managed to clarify this question by demonstrating different domains of the protein being required for the synthesis of membrane organelles of DENV. ${ }^{[3,24]}$ This output provides useful information in designing and determining peptide interventions with the property to bind on these domains to inhibit viral replication as a whole. Thus, via binding of those domains, the synthesis of DENV essential proteins is inhibited hence disrupting the required ecosystem for the virus to replicate effectively. Moreover, akin to the mechanism of an enzyme, the individualized peptide made for this intervention is not easily degradable thus enabling it to be used multiple times in succession. ${ }^{[3,24]}$ 
Akey et al. further expanded on this idea by identifying different potential binding domains in the various structural forms of the protein, especially in its dimer form. ${ }^{[8]}$ In Figure 1, the NS1 protein is presented in its dimer form with its three binding domains: the hydrophobic $\beta$-roll (blue), the wing that consists of $\alpha / \beta$ subdomain (yellow), and the $\beta$-ladder domain (red). Despite the $\beta$-ladder having minimal structural functionality, the hydrophobic $\beta$-roll plays an important role in the dimerization process of the protein while the wing domain is responsible to connect all the domains together into an integral structure. ${ }^{[18,6-8]}$ Hence, in theory, there is a possibility to design certain peptides that are complementary to these domains with the purpose in intercepting their physiology. ${ }^{[1]}$ Similar methods have been implemented by Huang et al. in which peptide-based antimicrobial agents are used on non-structural proteins. In this study, three major pathways were tested: the inhibition of viral entry, immunomodulation of the viral strain, and the interception of detailed steps on the viral replication cycle, with the latter being the most effective in reducing viral titre in the infected cell. ${ }^{[20]}$ The strategies were implemented on nonstructural proteins that plays a role in viral replication such as NS2B/NS3 (protease), NS5 (RNA-dependent RNA polymerase, RdRp), and NS3 (helicase) being the prime targets. ${ }^{[13,2]}$

Although NS1 proteins have yet to be targeted due to its enigmatic role in viral replication, there are a few properties of the protein that alleviate its status as a therapeutic agent compared to current interventions. Firstly, one of the biggest hurdles of vaccine development against dengue is due to the many serotypes of DENV. This translates to multiple vaccines needing to be tailored for each serotype and thus slowing the overall development. This is tackled by the housekeeping nature of NS1 protein, meaning that it exists in every serotype of DENV. Thus, treatment made against NS1 in theory would be effective regarding the DENV serotype. Secondly, previous peptide-based treatment targets structural proteins of DENV such as the envelope protein. These interventions named "neutralizing antibodies' 'block the attachment and entry of the virus into host cells. Albeit it being successful in doing so, they impose the risk of antibody dependent enhancement (ADE) in which viral infection is induced by antibody production of the host cell. This concern is tackled by the non-structural nature of NS1. Instead, antibodies made against NS1 or anti-NS1 showed a therapeutic effect in some studies, reducing viral replication by complementary binding to complementdependent cytotoxicity (CDC) of infected cells. ${ }^{[22-25]}$ Hence in short, the NS1 protein is a potent target for intervention due to it being highly conserved among genomes of different DENV serotypes thus one individualized peptide, in theory, should be effective in inhibiting viral replication albeit the DENV serotype. And secondly, peptide-based intervention would not elicit ADE thus increasing its applicability biosafety wise.

Peptide-Based Interventions on Viral Proteins of DENV

Antiviral peptides of DENV infection development focus on blocking virus entry of viral structural 
proteins function and enzymes. Furthermore, it blocks viral RNA processing host cells. ${ }^{[3]}$ Peptides drugs roles as inhibitors of viral replication, with the main targets being host cell receptors or attachment factors, viral structural proteins, and viral non-structural proteins. The drugs will prevent the attachment and binding of the viral proteins and subsequent entry of DENV. Furthermore, it will inhibit viral fusion and viral entry by targeting viral structural proteins. Modified peptide drugs that direct non-structural proteins will restrain the viral replication cycle. ${ }^{[13]}$

Antiviral peptides have several limitations. The major problems are the peptide's instability and bioavailability. In vivo activities shown as unmodified peptides in human serum degrade in a short time. There are numerous chemical modifications for increasing the peptides' stability and bioavailability by controlling the physicochemical parts of peptides. There are various ways to enhance the peptides' antiviral, such as identifying vital amino acids for inhibition by mutagenesis assays and cell-penetrating peptides and increasing cell permeability. To settle the oral bioavailability, it uses peptide sequence associated with cellpenetrating peptides (CPP) to deliver antiviral peptides for inhibitory activity by crossing the permeability barrier into the cell. Galactoside conjugated by tar protein can deliver the fusion protein to all the tissues in mice without restrained. ${ }^{[13]}$

\section{Efficacy Analysis of Peptide-Based NS1 Inhibitor from Current Studies}

Limited studies were available that directly analyzed the efficacy of peptide-based drugs on NS1 protein due to the only recent clarification of its function within the viral replication cycle. Despite that, there are two studies conducted by Songprakhon et al and Chen et al that analyzed the protein via in vivo and in vitro respectively. In Songprakhon et al, 11 potential peptides were identified using biopanning assays which were then further screened in silico using molecular docking to yield 4 sequences. These 4 sequences were then transferred into Huh7 cells that were infected by DENV-2 and viral production was then taken as a comparator to establish the protein's potential. Results were shown in Figure 5. With all potential peptides showing significant reduction in viral production in comparison with the untreated control. Furthermore, increasing the administration dose from $10 \mu \mathrm{M}$ to $20 \mu \mathrm{M}$ improves the reduction of viral production, concluding a dosedependent relation between inhibition of viral production and NS1 peptide concentration. ${ }^{[20]}$. From Chen et al., different in vivo studies of peptide-based intervention for the inhibition of NS1 protein were evaluated. Most studies yielded positive results with the outcome being improved survivability of the infected mice with DENV. Moreover, the peptide-based intervention also prevented hemorrhage due to severe DENV and reduced viremia within the mice. ${ }^{[20]}$ 

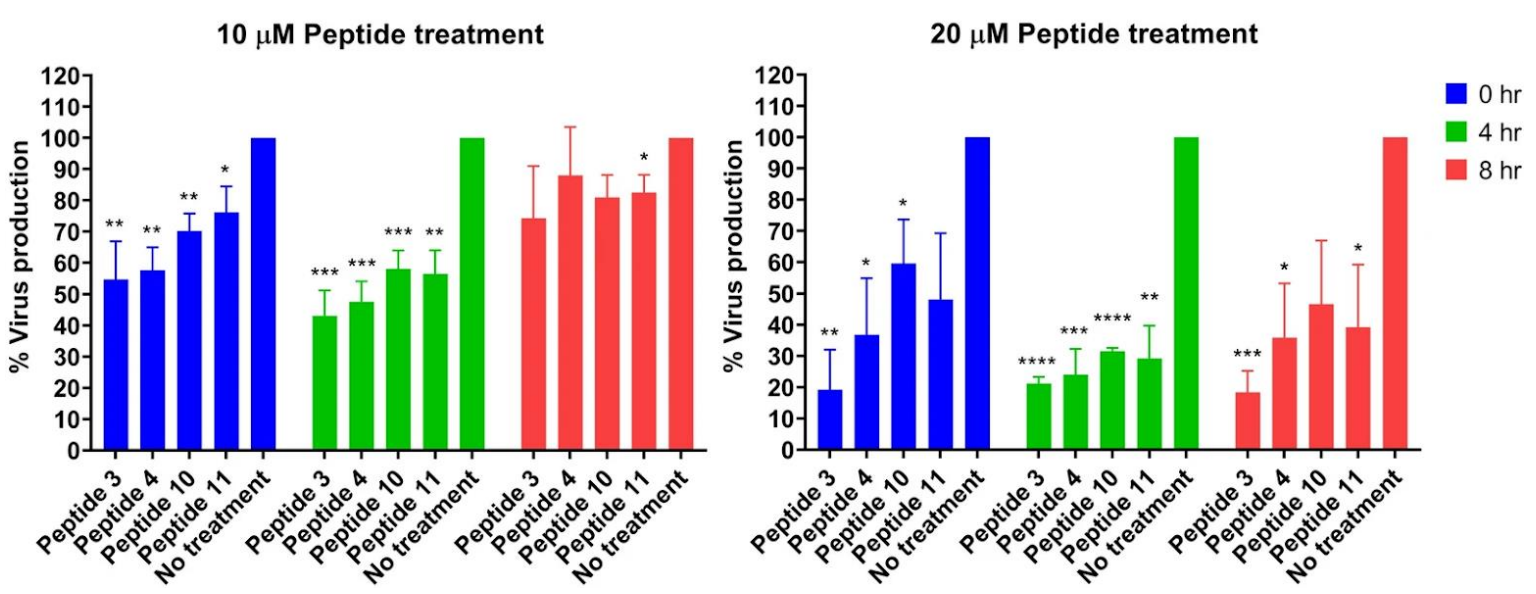

Figure 5. Viral Production Values after Peptide Intervention ${ }^{[20]}$

\section{Biosafety of Peptide-based Intervention}

Castanospermine, a natural alkaloid derived from Castanospermum Australae tree, has an ability to inhibit $\alpha$-glucosidase I and II. These enzymes were important in N-glycosylated glycoprotein folding mechanism. ${ }^{[24]}$ Celgosivir, a prodrug from castanospermine has been tested in phase 1 and 2 clinical trials in some HIV and HCV patients. However, there is no significant result in HIV and HCV cases yet having no significant side effects. ${ }^{[25]}$ Study regarding the celgosivir effect on another flavivirus, the DENV, has been tested by Low JG, et al in 2014. The results in this double-blinded randomised clinical trial were similar in HCV and HIV studies. Nevertheless, there are some mild-to-moderate diarrheas but no other severe effects reported in this study which mean Celgosivir is barely safe to use. ${ }^{[25]}$

\section{Strength and Limitations}

The strength of this literature review lies on the positively significant result of peptide-based interventions as a potential inhibitor of NS1 proteins. The values shown by Songprakhon et al were significantly impressive in comparison to the control culture. ${ }^{20}$ In vivo wise, the study conducted by Chen et al showed nearly all types of peptide approaches reaching a $100 \%$ survivability rate. ${ }^{23}$ Moreover, data from Table 1. shows the current vaccine update for DENV with most still being in phase $1 .{ }^{23}$ Hence, the urgency for an alternative treatment for DENV persists until a fully developed vaccine is publicly announced. ${ }^{27}$ However, due to the only recent understanding of the essentiality of NS1 protein, limited studies were available that could be included within this discussion. Furthermore, despite the identification of the essential role of NS1 protein, efficacy was only analysed via in vitro and in vivo. Hece, further clinical trials should be initiated to establish the efficacy and the effectiveness of peptide-based interventions on human subjects and control. 
Table 1. Current Vaccines for Dengue Status ${ }^{[23]}$

\begin{tabular}{|c|c|c|c|}
\hline No & Strategy & Developer & Current status \\
\hline 1 & $\begin{array}{l}\text { Live attenuated yellow fever } \\
\text { 17D/DENV chimeric vaccine }\end{array}$ & Sanofi-Pasteur & $\begin{array}{l}\text { Phase } 3 \text { trials with tetravalent } \\
\text { formulation in DENV endemic } \\
\text { countries }\end{array}$ \\
\hline 2 & $\begin{array}{l}\text { PDK cell-passaged live attenuated } \\
\text { vaccine }\end{array}$ & WRAIR/GSk & $\begin{array}{l}\text { Phase } 2 \text { trials with a tetravalent } \\
\text { formulation in endemic countries }\end{array}$ \\
\hline 3 & $\begin{array}{l}\text { Live attenuated DENV Delta-30 } \\
\text { mutation and intertypic DENV chimeric } \\
\text { vaccines }\end{array}$ & $\begin{array}{l}\text { NIH/Johns } \\
\text { Hopkins }\end{array}$ & $\begin{array}{l}\text { Phase } 1 / 2 \text { trials with monovalent } \\
\text { formulations completed; tetravalent }\end{array}$ \\
\hline 4 & Dengue prM-E DNA vaccine & NMRC & Phase 1 initiated \\
\hline 5 & $\begin{array}{l}\text { Recombinant } 80 \% \text { subunit antigen } \\
\text { vaccine }\end{array}$ & $\begin{array}{l}\text { Hawaii } \\
\text { Biotech/Merck }\end{array}$ & $\begin{array}{l}\text { Phase } 1 \text { with monovalent vaccine } \\
\text { initiated }\end{array}$ \\
\hline 6 & Purified inactivated vaccine & Wrair & $\begin{array}{l}\text { Phase } 1 \text { with monovalent vaccine } \\
\text { initiated }\end{array}$ \\
\hline 7 & Live attenuated chimeric DENV vaccine & $\mathrm{CDC}$ & $\begin{array}{l}\text { Phase 1 with monovalent vaccine } \\
\text { initiated }\end{array}$ \\
\hline
\end{tabular}

Abbreviations: PDK, primary dog kidney cells, WRAIR, Walter Reed Army Institute of Research; GSK, GlaxoSmithKline Biologicals; NIH. National Institute of Health; prM-E, pre-membrane-envelope; NMRC, Naval Medical Research Center; CDC, Centers of Disease Control and Prevention

\section{Conclusion}

In conclusion, DENV has been a lingering problem globally especially in developing and tropical countries. Despite having much focus being put on the disease beforehand, the possibility of developing a vaccine has been futile due to the many serotypes of the virus. With the functional discovery of the NS1 protein, arose the possibility to implement peptide-based intervention that targets the protein. The protein plays a major role in the viral replication cycle, hence by inhibiting its role, transmission of the virus could be prohibited. In vitro and in vivo studies of the interventions yielded positive results in decreasing the viral concentration and improving the survivability rate of the subjects. Although, further in vivo trials and randomized controlled trials are recommended to ensure the optimal dose, administration method, and efficacy of the peptide intervention. Moreover, proper cost-effectiveness analysis should be conducted on the mass production of the peptide and a concrete plan of the public health intervention if deemed suited for an Indonesian context. Hopefully, the implementation of this novel intervention could reduce the burden of DENV as a neglected tropical disease thus contributing to the World Health Organization road map and sustainable development goals (SDG) number 3, which is to ensure good health and wellbeing especially in eradicating all infectious diseases by the year 2030 . 
Green Medical Journal Vol.3 Issue: 1 (April, 2021)

e-ISSN: 2686-6668

\section{Conflict of Interest}

The authors declare no competing interests.

\section{Funding Sources}

None were received

\section{Acknowledgement}

We would like to thank Asian Medical Students Association Universitas Indonesia (AMSA-UI) for the unending support and guidance throughout the synthesis of this paper

\section{References:}

1. Akey DL, Brown WC, Jose J, Kuhn RJ, Smith JL. Structure-guided insights on the role of NS1 in flavivirus infection. BioEssays. 2015; 37(5): 489-94. doi:10.1002/bies.201400182

2. Harapan H, Michie A, Mudatsir M, Sasmono RJ, Imrie A. Epidemiology of dengue hemorrhagic fever in Indonesia: analysis of five decades data from the National Disease Surveillance. BMC Res Notes. 2019; $12: 350$

3. Songprakhon P, Thaingtamtanha T, Limjindaporn T, Puttikhunt C, Srisawat C, Luangaram P, et al. Peptides targeting dengue viral nonstructural protein 1 inhibit dengue virus production. Sci Rep. 2020; 10: 12933

4. Płaszczyca A, Scaturro P, Neufeldt CJ, Cortese M, Cerikan B, Ferla S, et al. A novel interaction between dengue virus nonstructural protein 1 and the NS4A-2K-4B precursor is required for viral RNA replication but not for formation of the membranous replication organelle. PLOS Pathogens. 2019; 15(5): e1007736-. doi:10.1371/journal.ppat.1007736

5. Jacobs MG. Dengue virus nonstructural protein 1 is expressed in a glycosyl-phosphatidylinositol-linked form that is capable of signal transduction. The FASEB Journal. 2000; 14(11): 1603-10.

6. Kao Y-S, Yu C-Y, Huang H-J, Tien S-M, Wang W-Y, Yang M, et al. Combination of Modified NS1 and NS3 as a Novel Vaccine Strategy against Dengue Virus Infection. The Journal of Immunology. 2019 Aug 26;203(7):1909-17.

7. Modhiran N, Watterson D, Muller DA, Panetta AK, Sester DP, Liu L, et al. Dengue virus NS1 protein activates cells via Toll-like receptor 4 and disrupts endothelial cell monolayer integrity. Science Translational Medicine. 2015 Sep 9;7(304):304ra142-2.

8. Chen J, Ng MM-L, Chu JJH. Activation of TLR2 and TLR6 by Dengue NS1 Protein and Its Implications in the Immunopathogenesis of Dengue Virus Infection. Kuhn RJ, editor. PLOS Pathogens. $2015 \mathrm{Jul}$ 30;11(7):e1005053.

9. Glasner DR, Puerta-Guardo H, Beatty PR, Harris E. The Good, the Bad, and the Shocking: The Multiple Roles of Dengue Virus Nonstructural Protein 1 in Protection and Pathogenesis. Annu Rev Virol. 2018; 10-101416.

10. Scaturro P, Cortese M, Chatel-chaix L, Fischl W, Bartenschlarger R. Dengue Virus Non-structural Protein 1 Modulates Infectious Particle Production via Interaction with the Structural Proteins. PLoS Pathog. 2015 Nov 12; 11(11):e1005277.

11. Huang Y, Lee C, Wang T, Kao Y, Yang C, Lin Y, et al. The Development of Peptide-based Antimicrobial Agents against Dengue Virus. Curr Protein Pept Sci. 2018 Oct; 19(10): 998-1010. 
12. Chew MF, Poh KS, Poh CL.Peptides as Therapeutic Agents for Dengue Virus. Int J Med Sci. 2017; 14(13):1342-1359.

13. De La Guardia C, Lleonart R. Progress in the identification of dengue virus entry/fusion inhibitors. Biomed Res Int. 2014; 2014:825039.

14. Alen MM, Schols D. Dengue virus entry as target for antiviral therapy. J. Trop. Med. 2012; 2012:628475

15. Rokom. Data Kasus Terbaru DBD di Indonesia [Internet]. Kementerian Kesehatan RI; 2020 [cited 2021 Apr 18]. Available from: https://sehatnegeriku.kemkes.go.id/baca/umum/20201203/2335899/datakasus-terbaru-dbd-indonesia/

16. Bhakat S, Karubiu W, Jayaprakash V, Soliman ME. A perspective on targeting non-structural proteins to combat neglected tropical diseases: Dengue, West Nile and Chikungunya viruses. Eur. J. Med. Chem. 2014;87:677-702

17. World Health Organization. Dengue guidelines, for diagnosis, treatment, prevention and control. World Health Organization; 2009

18. Norazharuddin H, Lai NS. Roles and Prospects of Dengue Virus Non-structural Proteins as Antiviral Targets: An Easy Digest. Malays J Med Sci. 2018 Sep; 25(5): 6-15.

19. Cervantes-salazar M, Angel-ambrocio AH, Soto-acosta R, Bautista-carbajal P, Hurtado-monzon AM, Alcaraz-estrada S, et al. Dengue virus NS1 protein interacts with the ribosomal protein RPL18: This interaction is required for viral translation and replication in Huh-7 cells. Virology. 2015; 484: 113-26

20. Songprakhon P, Thaingtamtanha T, Limjindaporn T, Puttikhunt C, Srisawat C, Luangaram P et al. Peptides targeting dengue viral nonstructural protein 1 inhibit dengue virus production. Scientific Reports. 2020;10(1).

21. Stevens A, Gahan M, Mahalingam S, Keller P. The Medicinal Chemistry of Dengue Fever. Journal of Medicinal Chemistry. 2009;52(24):7911-7926.

22. Akey D, Brown W, Dutta S, Konwerski J, Jose J, Jurkiw T et al. Flavivirus NS1 Structures Reveal Surfaces for Associations with Membranes and the Immune System. Science. 2014;343(6173):881885.

23. Chen H, Lai Y, Yeh T. Dengue virus non-structural protein 1: a pathogenic factor, therapeutic target, and vaccine candidate. Journal of Biomedical Science. 2018;25(1).

24. Rathore APS, Paradkar PN, Watanabe S, Tan KH, Sung C, Connolly JE, et al. Celgosivir treatment misfolds dengue virus NS1 protein, induces cellular pro-survival genes and protects against lethal challenge mouse model. Antiviral Research. 2011;92(3):453-60

25. Low JG, Sung C, Wijaya L, Wei Y, Rathore APS, Watanabe S. Efficacy and safety of celgosivir in patients with dengue fever (CELADEN): a phase $1 \mathrm{~b}$, randomised, double-blind, placebo-controlled, proof-of-concept trial. The Lancet Infectious Diseases. 2014;14(8):706-15 